\title{
BIOMATERIALS USED AS BONE GRAFT SUBSTITUTES
}

S. Narang, V. Chava. Biomaterials used as bone graft substitutes. Annal Dent Univ Malaya 2000; 7: 36-42.

\begin{abstract}
The ultimate goal of periodontal therapy should not be limited to the establishment and maintenance of periodontal health. The potential for regeneration of the hard and soft periodontal tissues lost to disease should be considered. Of all the bone grafting materials being developed, the demineralized freeze dried bone allograft (DFDBA) has been used as a substitute for bone graft for more than four decades. The basis for the use of any bone grafting material is to induce bone formation. In this article various biomaterials used are reviewed.
\end{abstract}

Key words: Bone, Graft, Biomaterial.

\section{INTRODUCTION}

The upcoming of various bone grafts and biomaterials has given a big leap to the field of Periodontics. The aim of periodontal therapy is to control periodontal infection and inflammation in the periodontium. The continued function of the periodontally involved tooth requires additional periodontal support and regeneration of the lost periodontium $(1,2)$. This may be achieved by the use of guided tissue regeneration and various bone grafts and biomaterials used as substitutes of bone grafts. These are derived from vital or non vital bone sources or consist of organic or inorganic materials. The main aim of utilizing bone grafts and performing bone graft related osseous surgeries is to promote bone regeneration. However these materials should be biologically acceptable to the patient without causing any systemic or local hazards or side effects. Often, autogenous bone is not available in sufficient quantity and if available requires additional surgical sites as well as an increased amount of chair time for the patient. Allogeneic bone is an alternative to autogenous bone, but poses the fear of disease transmission. This prompted the development of various bone substitutes which have provided us biocompatible defect fillers with differing biologic activity (1).

\section{BASIC CONSIDERATIONS}

\section{Definitions:}

1. Graft:

A graft is a viable tissue that after removal from the donor site is implanted within the lost tissue which is then restored, repaired or regenerated (3).

Examples: Autografts and Allografts.
Review Article

S. Narang ${ }^{1}$ and V. Chava

'Post Graduate Student
Department of Periodontics
'Department of Periodontics
S.D.M. College Of Dental Sciences
And Hospital
Dharwad-580 009, Karnataka,
INDIA
Corresponding author - V. Chava

\section{Biomaterials:}

These are bone derived materials processed in such a way that the cells are no longer vital and hence used as bone graft substitutes (1).

Examples:

- Freeze dried bone, decalcified or nondecalcified.

- Xenograft from a bovine derived material can be more appropriately called Xenogenic bone derived biomaterial.

\section{Osteogenesis:}

Occurs when cells of graft survive the transplantation and contribute to the repair process. The term has been used by some authors to define bone grafts with the capability of forming bone through osteoblastic cells contained in the transplanted site $(2,3)$.

\section{Osteo conduction:}

Process also known as Trell's effect, occurs with the ingrowth of capillaries in the new connective tissues. A material is osteo conductive when its structure and its chemical composition facilitate new bone formation from existing bone. This can be seen with various bone grafts or non bone grafts such as synthetic materials $(1,3)$.

\section{Osteo induction:}

It is a process or a set of processes, which initiate osteogenesis through cells orginally not involved in bone formation (1).

Examples: A graft, a biomaterial or a substance is osteo inductive when implanted in a non osseous environment called as an ectopic site, bone formation occurs.

The Ideal Requirements for bone grafts or bone graft substitutes may be summarised as follows:
i. Biologic acceptability
ii. Predictability
iii. Clinical feasibility 
iv. Minimal operative hazard

v. Minimal post operative sequelae

vi. Patient acceptance.

\section{BONE GRAFTS AND BONE GRAFT SUBSTITUTES}

Figure I illustrate the categories of bone grafts and bone graft substitutes.

\section{I) Non-osseous material}

A) Organic

i) Dentin

Although demineralised dentin has been advocated as a possible graft (5), it has not gained widespread acceptance (6) because clinical results have been somewhat disappointing.

ii) Dura

The use of lyophilised allogeneic duramater in periodontal osseous defects has resulted in gain of clinical attachment and reduction in probing depth. Histologically bone has been seen alongside but never in the implant (7).

\section{iii) $\underline{\text { Sclera }}$}

Success has been reported with preserved sclera (4), but there were no signs of osteogenesis within the sclera and it remained encapsulated at the site (6).

\section{iv) Cartilage}

Cartilage allografts provide no statistically significant advantage in bone or soft tissue repair over flap debridement alone (9).

\section{B) Inorganic}

i) Plaster of Paris

Plaster of Paris (Calcium sulphate) is biocompatible and porous thereby allowing fluid exchange, which prevents flap necrosis. Plaster of Paris resorbs completely in 1-2 weeks(10). Also, because plaster of paris is so rapidly resorbed, it is questionable whether it is of any benefit.

\section{ii) Bio active glasses}

There are 2 forms of bioactive glass currently available, perio glass (Bio glass synthetic bone graft particulate) and biogran (resorbable synthetic bone graft). Bio active glasses are composed of $\mathrm{SIO}_{2}, \mathrm{CaO}, \mathrm{Na}_{2} \mathrm{O}, \mathrm{P}_{2} \mathrm{O}_{5}$ and bond to bone is through the development of a surface layer of carbonate hydroxyapatite(HA). When exposed to tissue fluids invivo, the bioactive glass is covered by a double layer composed of silica gel and a calcium phosphate-rich (apatite) layer. This calcium phosphate-rich layer promotes adsorption and concentration of proteins used by osteoblasts to form a mineralised extracellular matrix. It is theorized that these bioactive properties guide and promote osteogenesis, allowing rapid and quick formation of new bone (11).

\section{Bone derived material}

A) Intraoral autografts.

a) (i) Osseous coagulum and bone blend

Robinson, in 1969, having considered that the grafted particles of bone should be of smaller size and that mineralised fragments can induce osteogenesis, developed a technique, which he termed osseous coagulum, using the material evacuated during osseous surgery. The donor bone is obtained from any intra-oral source, usually areas requiring osseous correction. A number 6 or 8 round carbide bur, rotating at 25000 to $30000 \mathrm{rpm}$, without irrigation, produces bone dust which when coated with blood makes an osseous coagulum. Robinson showed the feasibility of the technique in several case reports with re-entries from 3 to 24 months. Diem et al in 1972, proposed the use of an amalgam trituration capsule and a pestle to reduce bone fragments to a workable, plastic like mass, called Bone Blend (1).

ii) Intra-oral cancellous bone marrow transplants.

Haitt and Schallhorn have described the use of cancellous bone obtained from the maxillary tuberosity, edentulous areas and healing sockets. The maxillary tuberosity frequently contains a good amount of cancellous bone, particularly if the third molars are absent. Foci of red marrow are also occasionally observed. Edentulous ridges can be approached with a flap, and cancellous bone and marrow are removed with curettes. Sockets are allowed to heal for 8-12 weeks, and the apical portion is utilised as donor material. The particles are reduced to small pieces (4).

b) Bone swaging.

This technique requires the existence of an edentulous area adjacent to the defect, from which the bone is pushed into contact with the root surface without fracturing the bone at its base (4)

\section{B) Iliac bone and marrow autografts.}

Schallhorn, in 1968, described the first autogenous iliac bone graft in one case report where a bone marrow biopsy was immediately implanted into interproximal bony craters between both lower canines and lateral incisors. At 5 months re-entry, the defects appeared completely filled. Autografts of iliac bone and marrow possess a high osteogenic potential, even in supra crestal bone fill and class II furcations. They are able to induce cementogenesis, bone regeneration and Sharpey's fibres reattachment with functionally oriented periodontal ligament. Fresh iliac bone and marrow are responsible 
for a high rate of resorption and ankylosis. The need for an extra oral surgical site and for a sampling technique performed by a trained specialist represent serious limitations to this procedure (1)

\section{Allografts}

Iliac autografts have proven to be a predictable osseous grafting material, however, this necessitates an additional extra oral surgical procedure in order to obtain the grafting material. Allogeneic bone eliminates the need for additional surgery (1). Attempts have been made to suppress the antigenic potential of allografts and xenografts by radiation, freezing and chemical treatment. Bone allografts are commercially available from tissue banks. They are obtained from cortical bone within 12 hours of the death of the donor, defatted, cut in pieces, washed in absolute alcohol, and deep frozen. The material may or may not then be demineralised and subsequently, it is ground and sieved to a particle size of 250 to $750 \mathrm{~mm}$ and freeze dried. Finally it is vaccum sealed in glass vials (4).

In conclusion, allografts do not elicit any adverse immune response; have the same potential for regeneration as iliac autografts: crestal bone regeneration and regeneration of a normal periodontium have been demonstrated, and they do not give rise to root resorption or ankylosis. However, this approach, in spite of its many advantages, is not recommended because of the high risk of disease transmission.

\section{BIOMATERIALS}

Figure II illustrates the categories of Biomaterials.

\section{Bone derived biomaterials}

\section{A. Freeze dried human bone}

These are bone graft substitutes which are available through bone banks and are obtained from autopsy of human donors within 24 hours after death and are further lyophilized after a series of physical and chemical processing. In this procedure the guide lines of the American Association of Tissue Banks is followed (1).

It includes:

- Bone procured from the human donor within 24 hours after death.

- It is frozen to $-179^{\circ} \mathrm{C}$ for 4 weeks.

- Graft is freeze dried.

- Graft is defatted in chloroform menthol for 6 hours at $25^{\circ} \mathrm{C}$.

- Autodigested in phosphate buffers containing iodoacetate acid and sodium azide for 72 hours at $37^{\circ} \mathrm{C}$

- Demineralized in $0.6 \mathrm{~N} \mathrm{HCL}$ for 3 days at $4^{\circ} \mathrm{C}$.

- After each step bone is washed with triple distilled water.

- Refrozen and freeze dried.

- Ground under cryogenic conditions to yield a particle size of 125-1000 microns.

Bone grafts and Bone graft substitutes

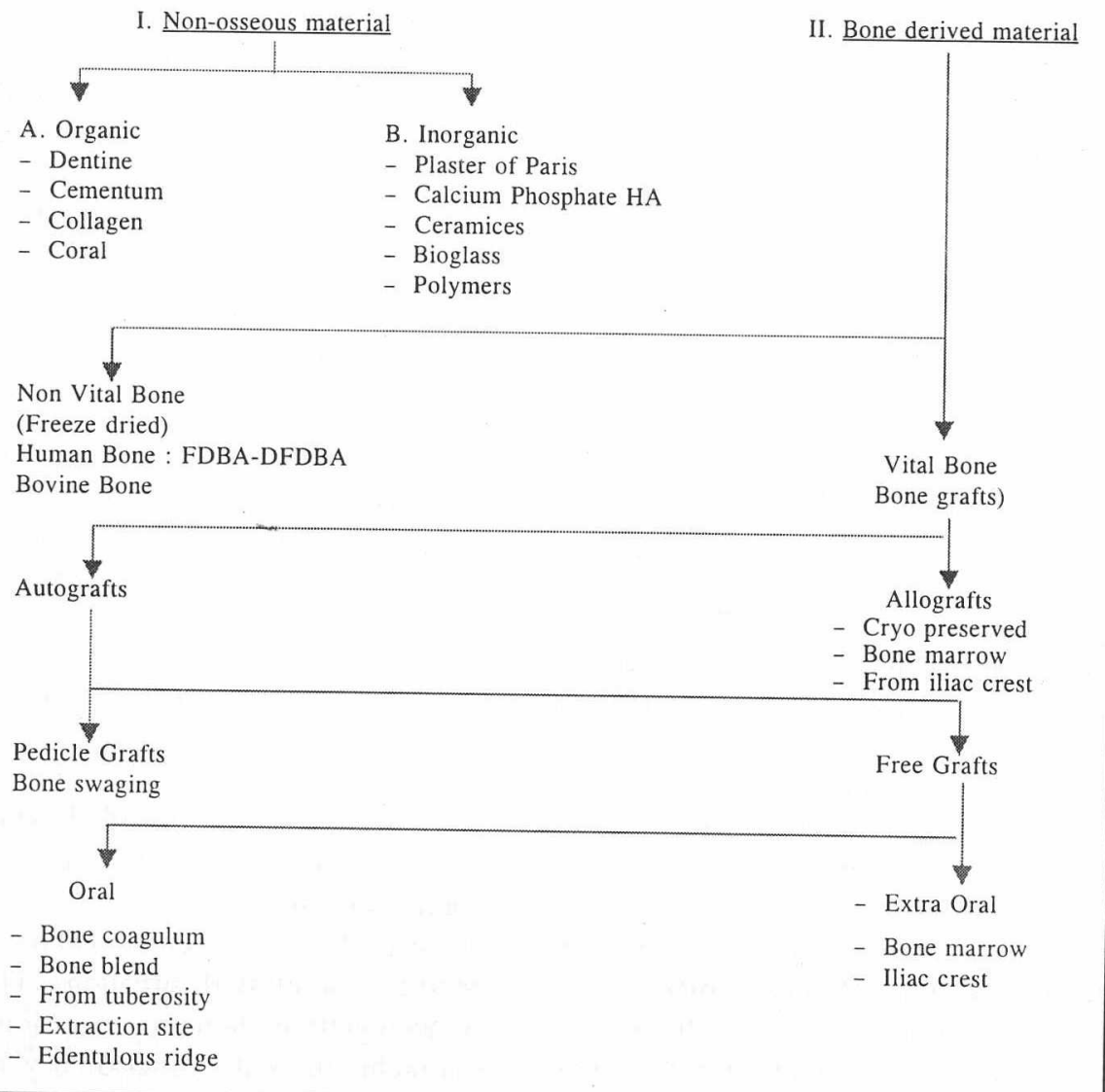

Figure 1: Categories of bone grafts and bone graft substitutes 
Biomaterials

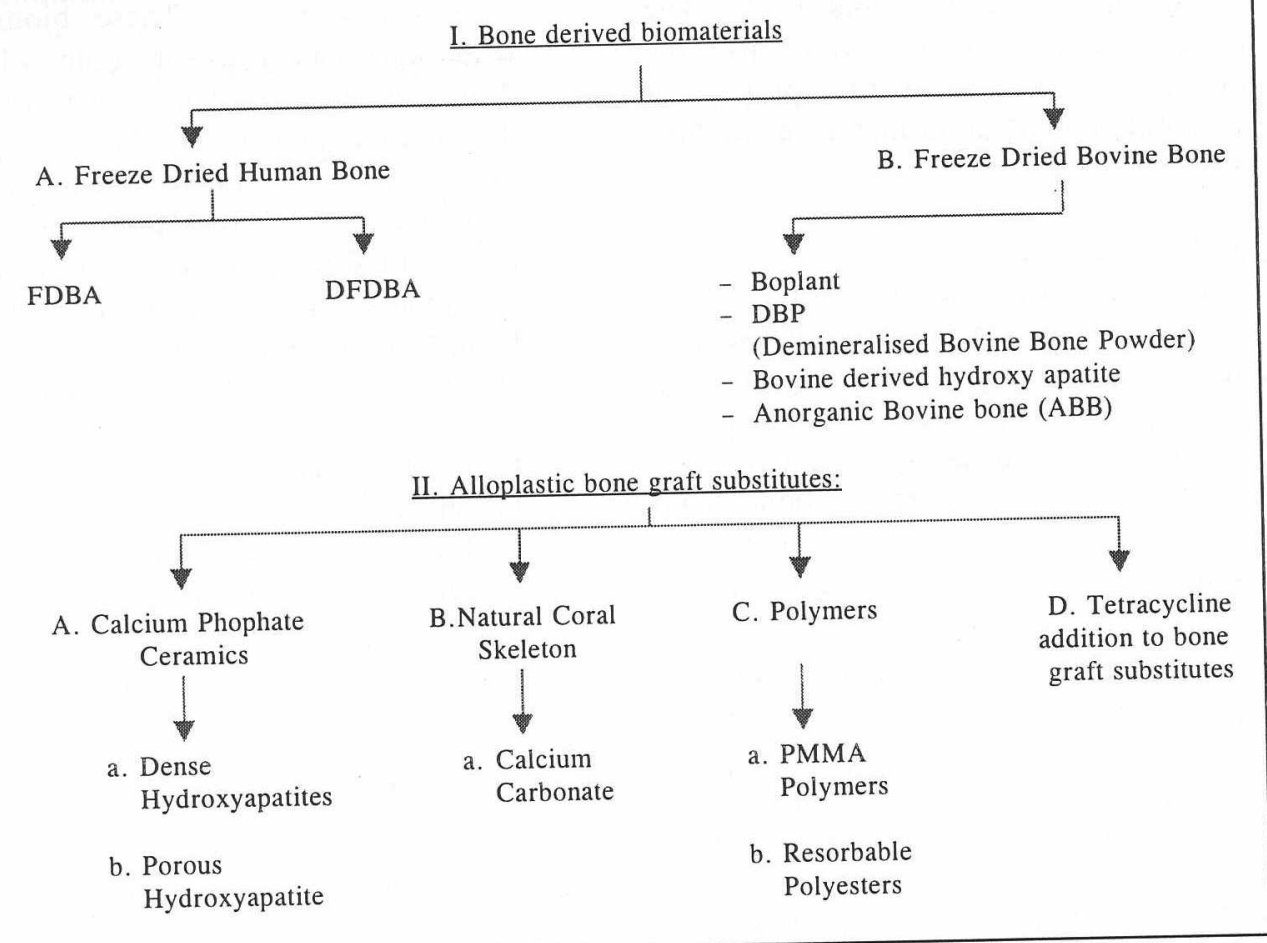

Figure 2: Categories of Biomaterials.

I. Bone derived. II. Alloplastic bone derived substitutes.

The osteo inductive capacity of these materials is governed by the processing procedure. The inductive activity gradually decreases and eventually is reduced to 0 within a period of 15 days when decalcification with $0.6 \mathrm{~N} \mathrm{HCL}$ is performed at $25^{\circ} \mathrm{C}$. wheareas in cold temperature $\left(2^{\circ} \mathrm{C}\right)$, the inductive activity is fairly maintained even at 30 days. Ethyl or isopropyl alcohols in $0.6 \mathrm{~N} \mathrm{HCL}$ produces total inactivation of inductive substitute. Delipidization with ether, alcohol, acetone, hexachlorophene, common detergents and Tween 80 may even enhance bone induction. Heating above $60^{\circ} \mathrm{C}$ inhibits bone formation.

\section{B. Freeze Dried Bovine Bone}

i) Boplant:

The use of Boplant, a bovine bone processed by detergent, chloroform and methanol extraction to reduce lipid content, washed, sterilized in antiseptic solution and lyophilized was first used way back in 1966 (1). The extensive usage of this material led to the conclusion that the material was incorporated by new bone formation, and it was accepted as a well tolerated, non resorbable, osteoconductive material. It was observed that the osteogenic potential of decalcified Boplant did not appear greater than that of mineralized Boplant $(1,12)$.

ii) Demineralized bovine bone powder (DBP) It is prepared from cortical portions of bovine bone after 1 hour extraction in $100 \%$ ethanol and ethyl ether. It is then pulverized in liquid nitrogen mill, demineralized for 3 hours in $0.5 \mathrm{~N} \mathrm{HCI}$ and washed with water, absolute ethanol and anhydrous ether until neutral pH is achieved (1).

iii) Bovine derived Hydroxyapatite

This is a xenogenic bone graft substitute, described as $100 \%$ crystalline, naturally porous, bovine derived hydroxyapatite. The use of this material was reported in two cases by Callan and Rohrer, in 1993(1).

iv) Anorganic bovine bone $(\mathrm{ABB})$

This is also a xenogenic variety, said to contain carbonate apatite. It is defatted and collagen is eluted. Though various studies were conducted by Thaller et al in 1993 and 1994 on animal models, there has been no study testing $\mathrm{ABB}$ in human periodontal defects to date (1).

\section{Risks of disease transmission}

Transmission of blood borne and tissue borne disease has raised concern about allogenic bone graft substitutes $(1,13)$. The xenogenic bone has also recently been suspected of potentially transmitting prion diseases. AIDS and Creutzfeldt - Jakob diseases (CJD) are at risk of getting transmitted by allotransplantation and bovine spongiform encephalopathy (BSE) could get transmitted by bovine bone transplantation. Both BSE and CJD belong to a group of diseases affecting CNS in animals as well as in humans. Scrapie is the prototype of these diseases. It affects mainly sheep, while BSE, a new form of prion disease, also known as mad-cow disease, affects bovines. In human CJD, Gerstmann - Straussler 
- Sheinker (GSS) disease and Kurv represents the main form of this disease. These are characterized by excitability, itching, ataxia, paralysis and death. The diagnosis of this disease is by autopsy. At autopsy, the brain show spongiform changes, formation of amyloid plaques and accumulation of abnormal form of host encoded proteins.

\section{Prevention of CJD transmission:}

There is not, till date, any marker of CJD contamination to screen potential donors because only brain post mortem biopsy can identify degenerative encephalopathies (1). Even the most stringent donor screening cannot assure the exclusion of donors with prepatent CJD. The World Health Organisation(WHO) has recommended 3 methods of sterilization, though total inactivation is not warranted (1). They are as follows:

a. Auto claving at $134^{\circ} \mathrm{C}$ to $138^{\circ} \mathrm{C}$ for 18 minutes or at $132^{\circ} \mathrm{C}$ for 1 hour.

b. Sodium hydroxide solution, $1 \mathrm{M}$ for 1 hour at $20^{\circ} \mathrm{C}$.

c. Sodium hypochlorite (at least $2 \%$ of chlorine) for 1 hour at $20^{\circ} \mathrm{C}$.

Until now no case of CJD transmission via bone derived biomaterial has been reported. However, one has to bear in mind that the mean latency of appearance of initial symptoms after iatrogenic CJD transmission is 59 months.

\section{Prevention of HIV transmission:}

HIV can be transmitted to patients receiving kidney, heart and liver transplants, as well as skin grafts and bone grafts. Rigorous screening of potential donors by medical and social screening, HIV antibody test, HIV antigen test, autopsy and special lymph node examinations is critical. Buck et al 1989, have calculated that the risk could be as low as $0.00006 \%$ (1). Mellonig et al 1992, have demonstrated that HIV infected bone graft is rendered free of virus contamination after the DFDBA processing (1).

\section{Alloplastic Bone Graft Substitutes \\ A. Calcium phosphate ceramics.}

The currently used calcium phosphate compounds are hydroxyapatite (HA) [Ca10 (Po4)6(OH)2] and beta tricalcium phosphate ( $\beta$-TCP) [Ca3)PO4)2]. These are solid biomaterials obtained from powdery phosphates. First, compression with slowly increasing pressure reduces the inter particle spaces, followed by a sintering process at a temperature of $1100^{\circ} \mathrm{C}$ to $1500^{\circ} \mathrm{C}$ (Osborn and Newesely, 1980) (1). A porous hydroxyapatite is also obtained by a hydrothermal exchange process in which natural coral calcium carbonate of genus porites is converted into hydroxyapatite (14).

\section{a. Dense Hydroxyapatite: (HA)}

The usage of this material has been widespread and the ultra structural mechanism of bone formation with various bioceramics implanted in periodontal tissues has been described. It has been observed that the smaller the size of the ceramic, the better the bone formation (15). These biomaterials are non resorbable bone graft substitutes when used in their dense polycrystalline form (16). Some bio degradation may occur when preparation of HA material gives rise to amorphous or porous structure. Though the clinical use of this material has resulted in reduction in probing depth, clinical attachment gain and partial bone defect resolution, human biopsies have shown no new periodontal attachment, osteogenesis or cementogenesis (1).

b. Porous hydroxyapatite (PHA)

Porous hydroxyapatite has shown results similar to DFDBA and FDBA and it has been observed histologically in humans and animals that PHA is unable to regenerate a connective tissue attachment. Whether PHA is able to enhance bone formation in an orthotopic site is controversial $(1,17)$.

c. $\beta$-Tricalcium phosphate:

It is produced from calcium phosphate powder mixed with napthalene, pressed and heated at temperatures as high as $10000^{\circ} \mathrm{C}$ to $20000^{\circ} \mathrm{C}$ allowing it to fuse in a solid mass. The napthalene evaporates, leaving spaces within the ceramic giving rise to a porous structure (1). It is difficult to ascertain if $\beta$-TCP is of any benefit in treating periodontal osseous defects. Histologically, no osteogenesis or reattachment has been truly demonstrated (18).

\section{B. Natural Coral Skeleton (NCS) - (Calcium Carbonate)}

It is a bone graft substitute of a genus porites, obtained from the Great Barrier Coral Reef in New Caledonia. After gathering, it is sundried for a few days, then organic parts are cleaned by ultrasonic treatment and sterilized in an autoclave at $131^{\circ} \mathrm{C}$ for $60 \mathrm{~min}$. This coral skeleton consists of calcium carbonate (99\%) in the form of arganite, the remaining $1 \%$ being organic nutrient amino acids. It is porous in nature with the average pore diameter of $150 \mu \mathrm{m}$. NCS has no bone inductive potential and the type of periodontal regeneration obtained by NCS is unknown as no human histology is available till date (1).

\section{Polymers:}

a. PMMA Polymers

This is a bone graft substitute, well tolerated by the recipient site. It is a combination of poly-methyl methacrylate (PMMA) and poly-hydroxyl-ethyl methacrylate (PHEM). The PMMA 550-580 mm diameter beads are embedded into PHEM and coated by calcium hydroxide. It has been shown to give favourable results when implanted in intra bony defects. But histologically, PMMA does not promote new attachment. PMMA has also been shown to have no osteoinductive property, but has been shown to possess osteoconductive properties in favourable bony defects (1). 
b. Resorbable Polyesters

The most commonly used polymers in medical applications are the poly hydroxy acids and particularly polymers with lactic acid (PLA) or glycolic acid (PGA). Many polymers can be synthesized from PLA. PLA and PGA vary in characteristics. PLA is highly resistant but brittle whereas PLG/PGA 50:50 posesses high elasticity. It has been observed that these polymers do not seem to be adapted to bone grafting. However their potential for bioresorption and ease of handling has encouraged some authors to use these biomaterials as carriers for osseopromoting agents or as linkers for other bone graft substitute. At present they are widely used as resorbable membranes in guided tissue regeneration (1).

D. Tetracycline Addition to Bone Graft Substitute:

It has been seen that local application of tetracycline (TTC) or doxycycline, combined with a bone graft substitute (FDBA or TCP)enhances bone periodontal regeneration in animal models. It has also been observed in humans that the addition of TTC to FDBA along with systemic administration of TTC enhances bone formation in juvenile periodontitis. However. it is not clear if the local application of TTC or doxycycline along with biomaterials other than FDBA has the same beneficial effects in adult or rapidly progressive periodontitis $(1,19)$.

\section{Fate of Graft Material:}

Once the material is placed in the bony defect it may act in a number of ways which may decide the fate of the graft material $(2,20)$. The various possibilities include:

Bone graft material may have no effect at all.

The bone graft material may act as a scaffolding material for the host site to lay new bone.

The bone graft material may itself deposit new bone because of its own viability.

\section{SUMMARY AND CONCLUSION}

From this review it is evident that bio materials used in Periodontics have shown remarkable results as compared to the simple procedures like open curettage, and flap debridement surgery alone for periodontal osseous defects. At present, barrier techniques to prevent epithelial downgrowth, with or without autogenous bone or decalcified freeze-dried bone allograft, are widely used. Coral - derived materials can be used when non human derived grafts are preferred. Bone substitutes will definitely have an increased role to play in the future of periodontal regeneration.

\section{REFERENCES}

1. Jean-Pirr Olika Youn. Proceedings of the $2^{\text {nd }}$ European workshop on Periodontics. In Niklous P.
Lang, ed. Bone grafts and biomaterials used as bone graft substitutes. Berlin, Quintic ssenz Verlag, 1997-313.

2. Polson A.M. Periodontal regneration Current status and directins. $1^{\text {st }}$ edition; Quintessence publishing Co., Inc 1995, 9-10: 71-112.

3. Grant, Stern, List garten, Periodontics. (Sixth edition). The C.V. Mosby Company 1988; 838-82.

4. Carranza F.A. and Newman M.G. Clinical periodontology, eighth edition, W.B. Saunders Company, 1996; 622-39.

5. Register, A.A. et al. Human bone induction by allogeneic dentin matrix. J Periodontol 1972; 43: 459.

6. Dragoo M.R. Clinical and histologic evaluation of alloplasts and allografts in regenerative periodontal surgery in humans. Int J Periodont Rest Dent 1983; 3(2): 8 .

7. Busschop J, and De Boener, J. Clinical and histologic characteristics of lyophilized allogeneic duramater in periodontal bony defects in humans. J Clin. Periodontol 1982; 9: 141.

8. Klingsberg J, Periodontal scleral grafts and combined grafts of sclera and bone: two year appraisal, J Periodontol 1974; 45: 262.

9. Chodroff, R.E. and Ammons, W.F. Periodontal repair and surgical debridement with and without cartilage allografts. J Clin. Periodontol 1984; 11 : 295.

10. Alderman NE Sterile plaster of Paris as an implant in the infrabony environment. A preliminary study. J Periodontol 1969; 40: 11.

11. Aichelmann - Reidy ME and Yukna R.A. Bone replacement grafts. The bone substitutes. Dent. Clin. North Am. Advances in Periodontics 1998; Part II 42(3): 491-501.

12. Mellonig, Bowers G.M. Comparison of bone graft materials. (Part I) J Periodontol 1981; 52: 291.

13. Sonis T, Williams L, Jeffcoat M. Healing of spontaneous periodontal defects in dogs treated with xenograft demineralized bone. J Periodontol 1985; 56: 470 .

14. Strub J.R., Gabertlvelt W. Comparison of tri calcium phosphate and frozen allogenic bone J Periodontol 1979; 50: 627.

15. Zaner D.T. Yukna R.A. Partial size of periodontal grafting material. J Periodontol 1987; 55: 406. 
16. Stahl S.S. Frowm S.J Histologic and clinical responses to porous hydroxy -lapatite implants in human periodontal defects J Periodontol 1981; 54: 589-695.

17. Oreameno S, Lekuvic V, Kenney E.B. Comparative clinical study of porous hydroxy apatite and DFDB. J Periodontol 1990; 61: 399.
18. Shimazaki K. Comparative study of porous Hydroxyapatite and Tri calcium phosphate. J of Orthopedic Research 1985; 3: 301-10.

19. Evan S, Yukna R.A., Sepe W W. Effects of various grafts materials with tetracycline in localized juvenile periodontitis. J Periodontol 1989; 60: 491.

20. Genco, Goldman, Cohen. Contemporary periodontic. $1^{\text {st }}$ Edition. The C.V. Mosby Company, 1990; 585-604. 